\title{
Vertragsentwicklung und Differenzierung in der europäischen Integration. Nationale Identität, staatliche Autonomie und die Entstehung einer Kern-Peripherie-Struktur in der Europäischen Union
}

\author{
Thomas Winzen und Frank Schimmelfennig*
}

Die Vertragsentwicklung der Europäischen Union lässt sich grob in zwei große Zeitabschnitte unterteilen. Die erste Phase vom Ende der 1950er bis zum Ende der 1980er Jahredie ,Gemeinschaftsphase ${ }^{6}$ - war einerseits durch eine weitgehende Stabilität des vertragsrechtlichen Rahmens gekennzeichnet, wie er durch die Römischen Verträge von 1957 vorgegeben war. Andererseits zeichnete sich die europäische Integration durch ein hohes Maß an Uniformität aus: Die Grundregeln der Europäischen Gemeinschaft galten für alle Mitgliedstaaten gleichermaßen - und ausschließlich für die Mitgliedstaaten. In der ,Unionsphase" seit Ende der 1980er Jahre zeigt sich hingegen eine sehr dynamische Vertragsfortschreibung: In kurzen Abständen folgten auf die Einheitliche Europäische Akte (1986) die Verträge von Maastricht (1992), Amsterdam (1997) und Nizza (2001), der - gescheiterte - Verfassungsvertrag (2004) und der Vertrag von Lissabon (2007). In dieser Phase hat sich die Integration zunehmend vertieft: Neue Politikfelder wie die Innen-, Außen- und Sicherheitspolitik sind hinzugekommen; zahlreiche Politikfelder (vor allem Binnenmarkt, Geld- und Währungspolitik, Asyl- und Migrationspolitik) wurden stärker vergemeinschaftet; das typische Entscheidungsverfahren entwickelte sich von Einstimmigkeit im Rat unter Konsultation des Europäischen Parlaments zu qualifizierter Mehrheit im Rat und Mitentscheidung des Parlaments.

In der gleichen Zeit hat sich die europäische Integration jedoch in hohem Maße differenziert: Zum einen gelten die vertraglichen Regelungen der Europäischen Union nun nicht mehr für alle Mitgliedstaaten im gleichen Umfang (interne Differenzierung), zum anderen gelten Teile der EU-Verträge der Sache nach auch in einigen Nicht-Mitgliedstaaten (externe Differenzierung). Das früheste und bekannteste Beispiel für eine dauerhafte interne Differenzierung ist die zwischen der Eurozone und dem Rest der Union. Externe Differenzierung zeigt sich besonders klar im Europäischen Wirtschaftsraum, der den europäischen Binnenmarkt um Island, Liechtenstein und Norwegen erweitert. Das Schengen-Gebiet mischt interne und externe Differenzierung, weil es Nichtmitglieder (wie Norwegen und die Schweiz) einschließt und Mitglieder (wie Großbritannien und Irland) ausschließt.

* Dr. Thomas Winzen, Center for Comparative and International Studies (CIS), Eidgenössische Technische Hochschule (ETH) Zürich.

Prof. Dr. Frank Schimmelfennig, Center for Comparative and International Studies (CIS), Eidgenössische Technische Hochschule (ETH) Zürich.

Dieser Artikel basiert auf dem EUDIFF1-Datensatz über die primärrechtliche Differenzierung in der Europäischen Union, der in einem von der Deutschen Forschungsgemeinschaft und dem Schweizerischen Nationalfonds geförderten gemeinsamen Forschungsprojekt der ETH Zürich (Leitung: Frank Schimmelfennig) und der Universität Konstanz (Leitung: Katharina Holzinger) erstellt wurde. Für weitere Informationen zu diesem Datensatz vgl. Frank Schimmelfennig/Thomas Winzen: Instrumental and Constitutional Differentiation in the European Union, in: Journal of Common Market Studies 2/2014, S. 354-370. Unser besonderer Dank gilt Nils-Christian Bormann (ETH Zürich) für seine Beratung bei der logistischen Regressionsanalyse. 
Schaubild 1 zeigt die unterschiedliche Rolle der differenzierten Integration in den zwei großen Phasen der Integrationsentwicklung deutlich. Während in der Gemeinschaftsphase nur etwa 1-2 Prozent der Vertragsartikel differenziert waren, ist der Anteil der differenzierten Vertragsartikel in der Unionsphase von etwa 10 Prozent nach dem Vertrag von Maastricht auf etwa 43 Prozent im Jahr 2013 angestiegen. Dabei ist nur die interne Differenzierung berücksichtigt. Nähme man die externe Differenzierung hinzu, dann müssten auch zahlreiche Vertragsartikel, die den Binnenmarkt und die Zollunion betreffen und für alle Mitgliedstaaten gelten, in den Anteil der differenzierten Artikel einfließen.

\section{Schaubild 1: Entwicklung der differenzierten Integration}

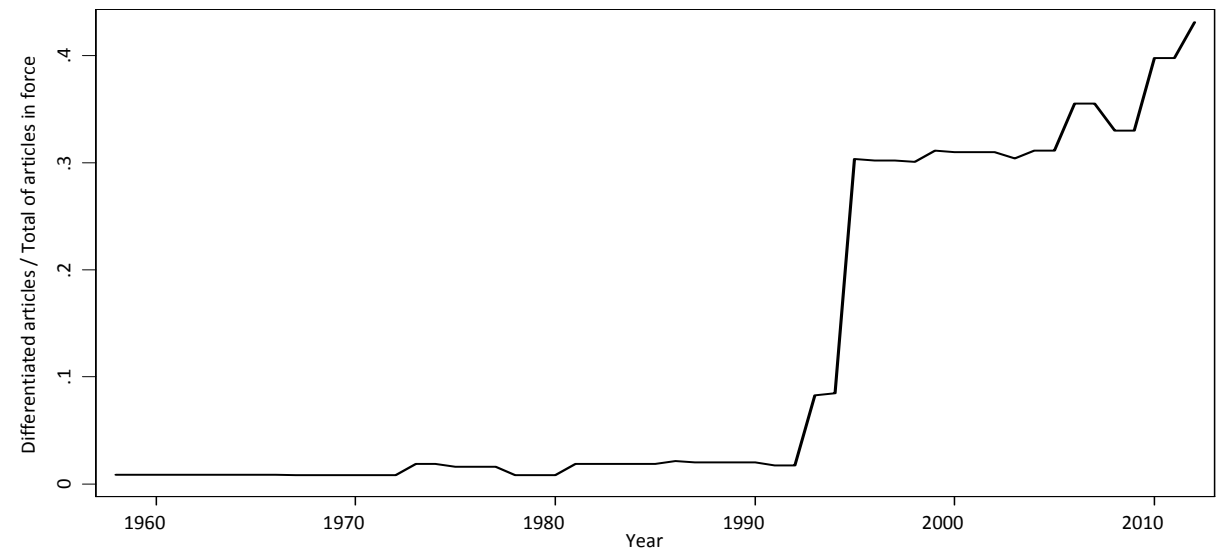

Anmerkung: Verhältnis von differenzierten Vertragsartikeln zur Gesamtheit der geltenden Vertragsartikel (ohne EGKS- und EAG-Verträge). Nur interne Differenzierung.

Quelle: Schimmelfennig/Winzen: Instrumental and Constitutional Differentiation, 2014.

Zusammenfassend lässt sich also sagen, dass Vertiefung und Differenzierung in der Integrationsentwicklung der vergangenen zwei Jahrzehnte Hand in Hand gingen. Dem Zusammenhang zwischen Vertragsentwicklung und Differenzierungsentwicklung wollen wir in diesem Beitrag nachgehen. Welche Faktoren begünstigen die Differenzierung? Welche Merkmale von Politikfeldern und Mitgliedstaaten sind für das Wachstum differenzierter Integration verantwortlich? In Übereinstimmung mit der konstruktivistischen Integrationstheorie argumentieren wir, dass differenzierte Integration eine Reaktion auf die Angst vor Autonomie- und Identitätsverlusten im Zuge der Vertragsentwicklung ist. Sie betrifft in erster Linie die Integration von staatlichen Kernkompetenzen und Mitgliedstaaten mit stark ausgeprägter nationaler Identität. Bereits bestehende Opt-outs, die Vertragsratifikation durch Volksabstimmungen und großer Wohlstand tragen ebenfalls zur differenzierten Integration bei. Wir zeigen diese Zusammenhänge anhand einer quantitativen Analyse der im EUDIFF1Datensatz enthaltenen vertragsrechtlichen Differenzierungen. Zunächst präsentieren wir unseren theoretischen Ansatz und unsere Hypothesen, dann unsere Daten und Variablen und schließlich die Ergebnisse einer logistischen Regressionsanalyse.

\section{Theoretische Perspektiven und Hypothesen}

In der Theorie europäischer Integration spielte die Differenzierung in der Vergangenheit nur eine Nebenrolle. Die großen Debatten in der Integrationstheorie zwischen Varianten des 
Neofunktionalismus und Intergouvernementalismus entzündeten sich an den zentralen Vertiefungsentscheidungen: der Entstehung der Europäischen Gemeinschaften, ${ }^{1}$ des Binnenmarktes und der Wirtschafts- und Währungsunion. ${ }^{2}$ Später wurden auch idealistische oder konstruktivistische Ansätze auf diese Vertiefungsentscheidungen angewandt. ${ }^{3}$ Die mit diesen Vertiefungsentscheidungen verbundene Differenzierung taucht in den Analysen aber nur als Nebenaspekt auf.

Generell wird differenzierte Integration in der politikwissenschaftlichen Literatur als Antwort auf die wachsende Heterogenität der Präferenzen und Kapazitäten der Mitgliedstaaten und Kandidaten verstanden, die im Zuge der fortschreitenden Erweiterung der Europäischen Union, ihres Ausgreifens in neue, kontroverse und politisierte Politikfelder und ihrer zunehmenden supranationalen Zentralisierung und Einschränkung nationaler Autonomie zutage tritt. Differenzierung ist unter diesen Bedingungen ein nützliches Instrument, um Entscheidungsblockaden zu umgehen und es den europäischen Staaten zu ermöglichen, in dem Umfang an der europäischen Integration beteiligt zu sein, der ihren Präferenzen und Fähigkeiten entspricht. ${ }^{4}$ Diese plausible Annahme lässt allerdings offen, welche mitgliedschaftlichen Präferenzen und Fähigkeiten für die differenzierte Integration ausschlaggebend sind und warum sich Mitgliedstaaten und Politikfelder hinsichtlich der Intensität differenzierter Integration stark unterscheiden.

Leuffen, Rittberger und Schimmelfennig formulieren daher ausgehend von den wichtigsten Integrationstheorien Hypothesen über differenzierte Integration auf der Basis von Staaten- und Politikfeldmerkmalen. ${ }^{5}$ Aus rationalistisch-intergouvernementalistischer Perspektive ist die Wahrscheinlichkeit von Differenzierung in solchen Politikfeldern besonders hoch, die von ausgeprägter materieller Heterogenität, also unterschiedlicher internationaler Interdependenz und unterschiedlichen ökonomischen Interessen der Staaten gekennzeichnet sind. Staaten mit geringerer internationaler Interdependenz, abweichenden Präferenzen und besonders hohen Anpassungskosten nehmen demnach am ehesten Opt-outs in Anspruch. Aus konstruktivistischer Perspektive hingegen sind die Heterogenität von Identitäten, Legitimitätsstandards und Politikideen sowie die innerstaatliche Politisierung ausschlaggebend. Staaten mit stark ausgeprägter nationaler Identität, geringer Legitimität und starker Politisierung der europäischen Integration sowie abweichenden Politikideen sollten demnach besonders stark von differenzierter Integration Gebrauch machen. In beiden Fällen verstärken hohe innerstaatliche Ratifikationshürden die Differenzierungswahrscheinlichkeit.

In einer früheren Analyse finden wir, dass die primärrechtliche Differenzierung in der Europäischen Union zwei unterschiedlichen Logiken folgt. ${ }^{6}$ Die ,instrumentelle Differenzierung', die im Kern mit den Annahmen des Intergouvernementalismus übereinstimmt, hat ihren Ursprung vor allem in den Übergangsbestimmungen, die in den Erweiterungsverträgen der Europäischen Union mit ihren neuen Mitgliedern vereinbart werden. Sie betrifft in erster

1 Vgl. Ernst B. Haas: The Uniting of Europe. Political, Social and Economic Forces 1950-1957, Stanford 1958; Alan Milward: The Reconstruction of Western Europe 1945-51, London 1984.

2 Vgl. Andrew Moravcsik: The Choice for Europe, Ithaca/New York 1998; Wayne Sandholtz: Choosing Union: Monetary Politics and Maastricht, in: International Organization 1/1993, S. 1-39; Wayne Sandholtz/John Zysman: 1992: Recasting the European Bargain, in: World Politics 1/1989, S. 95-128.

3 Craig Parsons: A Certain Idea of Europe, Ithaca/ New York 2003.

4 Katharina Holzinger/Frank Schimmelfennig: Differentiated Integration in the European Union: Many Concepts, Sparse Theory, Few Data, in: Journal of European Public Policy 2/2012, S. 292-305, hier S. 299.

5 Dirk Leuffen/Berthold Rittberger/Frank Schimmelfennig: Differentiated Integration. Explaining Variation in the European Union, Basingstoke 2013, S. 105-107.

6 Schimmelfennig/Winzen: Instrumental and Constitutional Differentiation, 2014. 
Linie den Binnenmarkt und die Wirtschaftspolitik der Europäischen Union. Zum einen versuchen Altmitglieder Ausnahmen zu erwirken, um die aus der Erweiterung resultierenden Belastungen für ihre Budgets, Produzenten und Arbeitnehmer zu verringern. Dies gilt insbesondere bei Umverteilungen zugunsten der Neumitglieder (vor allem in der Agrar- und Strukturpolitik) und bei der Öffnung der Arbeitsmärkte. Andererseits versuchen die Neumitglieder Wettbewerbsnachteile zu verzögern, die aus der Übernahme kostspieliger Produkt- oder Produktionsstandards (zum Beispiel bei Umweltauflagen) resultieren. In jedem Fall sind ökonomische Heterogenität und hohe Anpassungskosten die treibenden Kräfte der instrumentellen Differenzierung. Ärmere Neumitglieder, die besonders hohe Anpassungskosten und ein besonders hohes Potenzial für Arbeitsmigration und Subventionsansprüche besitzen, sind von instrumenteller Differenzierung am stärksten betroffen.

Die ,konstitutionelle Differenzierung ', die mit den konstruktivistischen Differenzierungshypothesen übereinstimmt, ist hingegen in erster Linie eine Folge von Revisionen der europäischen Verträge. Hier steht die Ausdehnung der europäischen Integration auf neue Politikfelder und die Übertragung von nationalstaatlichen Kompetenzen auf EU-Organe im Vordergrund. Solche Bestrebungen haben oft keine unmittelbaren ökonomischen Verteilungswirkungen, rufen aber integrationsskeptische Akteure auf den Plan, die sich um die Autonomie und Integrität des Nationalstaats sorgen. Nach dieser Logik betrifft die Differenzierung in erster Linie Politikfelder im Kernbereich staatlicher Souveränität, wie die innere und äußere Sicherheit und die Fiskalpolitik, sowie Mitgliedstaaten mit relativ stark ausgeprägter nationaler Identität. Außerdem vermuten wir, dass die ,konstitutionelle Differenzierung ‘ eher von den reicheren Mitgliedstaaten ausgeht. Diese Staaten können es sich besser ,leisten“, eine an Souveränitäts- und Identitätswahrung orientierte Politik zu verfolgen, als ärmere Mitgliedstaaten. Anders gesagt: Staaten sind eher bereit, Autonomie aufzugeben, wenn sie dafür relevante Wohlstandsgewinne erzielen oder Wohlstandsverluste vermeiden können. ${ }^{7}$

Diese aus einer deskriptiven Analyse resultierenden Erwartungen wollen wir nun weiter verfolgen und genauer testen. Da wir den Zusammenhang von Vertragsrevisionen und Differenzierung untersuchen, nehmen wir zunächst an, dass es sich um Fälle ,konstitutioneller Differenzierung' handelt. Auf Basis dieser Annahme formulieren wir die folgenden Hypothesen:

(1) Die Integration von staatlichen Kernkompetenzen ist mit höherer Wahrscheinlichkeit von Differenzierungen betroffen als die Integration von anderen Politikfeldern.

(2) Je stärker ausgeprägt die nationale Identität in einem Mitgliedstaat ist, desto wahrscheinlicher ist dieser Staat an differenzierter Integration beteiligt.

(3) Je wohlhabender ein Mitgliedstaat ist, desto wahrscheinlicher ist er an differenzierter Integration beteiligt.

Außerdem vermuten wir einen Interaktionseffekt zwischen Politikfeld und Identität. Zu erwarten ist, dass sich die nationale Identität vor allem bei der Integration von staatlichen Kernkompetenzen auswirkt, die für die nationale Identität und die nationalstaatliche Integrität von hoher Bedeutung sind.

(4) Die differenzierungsfördernde Wirkung nationaler Identität wirkt sich bei der Integration von staatlichen Kernkompetenzen stärker aus als in anderen Politikfeldern.

Schließlich nehmen wir an, dass hohe Ratifikationshürden und bestehende Differenzierungen die Wahrscheinlichkeit neuer Differenzierungen erhöhen. Die stärkste Ratifikationshürde für einen neuen europäischen Vertrag sind Volksabstimmungen. Sie erhöhen die

7 Walter Mattli: The Logic of Regional Integration: Europe and Beyond, Cambridge 1999. 
Wahrscheinlichkeit, dass sich euroskeptische Haltungen durchsetzen können, die in der breiten Bevölkerung im Allgemeinen stärker vertreten sind als auf Regierungsebene. Außerdem senken Opt-outs, die ein Staat in einem bestimmten Politikfeld bereits erhalten hat, die Hürde für neue Differenzierungen im selben Politikfeld.

(5) Ratifikation durch Volksabstimmung erhöht die Wahrscheinlichkeit differenzierter Integration.

(6) Bestehende Differenzierungen erhöhen die Wahrscheinlichkeit weiterer Differenzierungen für denselben Staat im selben Politikfeld.

\section{Daten und Variablen}

Wir sprechen von differenzierter Integration, wenn eine in Kraft getretene vertragliche Regel in mindestens einem Mitgliedstaat für mindestens ein Jahr keine rechtliche Gültigkeit besitzt. Diese Definition schließt (für alle Mitgliedstaaten gültige) Regeln aus, die weniger als eine vollständige Harmonisierung verlangen. Ebenso wenig gelten eine faktisch ungleiche Regeleinhaltung in den Mitgliedstaaten und die unterschiedliche Beteiligung an informeller mitgliedschaftlicher Kooperation als differenzierte Integration. Auch die sekundärrechtliche Differenzierung berücksichtigen wir hier nicht. Regeln sind zwar theoretisch gesehen die idealen Analyseeinheiten, in der Praxis aber oft nicht leicht zu identifizieren oder abzugrenzen. Auch sind sie nicht ohne Weiteres aus den formellen Unterteilungen der Verträge abzuleiten. Aus pragmatischen Gründen verwenden wir dennoch die Artikel der rechtlich verbindlichen Bestandteile europäischer Verträge (Hauptteil, Protokolle, Anhänge) als Analyseeinheiten für unseren Datensatz. Sie sind eindeutig zu identifizieren, regeln normalerweise einen abgegrenzten Sachverhalt und kommen damit der Idee einer einzelnen Regel immerhin nahe.

Für jeden Vertragsartikel verzeichnet der EUDIFF1-Datensatz unter anderem, ob eine Differenzierung vorliegt, ob es sich um eine potenzielle oder tatsächliche Differenzierung handelt (dürfen Mitgliedstaaten prinzipiell eine Ausnahme in Anspruch nehmen oder tun sie es tatsächlich?), in welchen Mitgliedstaaten der Artikel gilt beziehungsweise nicht gilt, in welchen Jahren dies der Fall ist und welches Politikfeld die Differenzierung betrifft. Damit lassen sich beispielsweise das Ausmaß der differenzierten Integration in jedem Jahr (siehe Schaubild 1), die Dauer einzelner Differenzierungen, die unterschiedliche Beteiligung und Betroffenheit der Mitgliedstaaten und das unterschiedliche Ausmaß der Differenzierung in den Politikbereichen der europäischen Integration beschreiben.

Für die Analyse des Zusammenhangs von Vertragsentwicklung und Differenzierung unterscheiden wir vier Zeitpunkte: das Inkrafttreten der Verträge von Maastricht (1994), ${ }^{8}$ Amsterdam (1999), Nizza (2003) und Lissabon (2010). ${ }^{9}$ Mit jedem Vertrag werden zusätzliche Politikfelder europäisch geregelt. Tabelle 1 führt diese Politikfelder auf. In einem weiteren Schritt fügen wir zu jedem Zeitpunkt die Mitgliedstaaten hinzu. Zum Zeitpunkt des Inkrafttretens des Vertrags über die Europäische Union (EUV) 1994 sind es beispielsweise 12 Mitgliedstaaten multipliziert mit 20 Politikfeldern, also 240 Beobachtungen; durch die Erweiterung auf 27 Mitgliedstaaten und die Zunahme der integrierten Politikfelder auf 28 wächst die Beobachtungszahl über Zeit an. Unter Berücksichtigung von Einschränkungen in der

8 Der Vertrag ist zwar 1993 in Kraft getreten, einzelne Bestimmungen aber erst 1994, darunter auch solche, die von Differenzierung betroffen waren.

9 Der Vertrag ist zwar schon am 1. Dezember 2009 in Kraft getreten, wird in unserem Datensatz aber erst ab dem Jahr 2010 geführt. 
Verfügbarkeit von Informationen zu unseren erklärenden Variablen, stehen für die Analyse somit insgesamt 1.893 Datenpunkte zur Verfügung.

Tabelle 1: Vertragsentwicklung, Politikfelder und Differenzierungen

\begin{tabular}{|c|c|c|c|c|}
\hline Politikfeld & EUV & Amsterdam & Nizza & Lissabon \\
\hline \multicolumn{5}{|l|}{ Prinzipien } \\
\hline \multicolumn{5}{|l|}{ Warenverkehrsfreiheit } \\
\hline \multicolumn{5}{|l|}{ Landwirtschaft } \\
\hline \multicolumn{5}{|l|}{ Personenverkehrsfreiheit } \\
\hline \multicolumn{5}{|l|}{ Niederlassungsfreiheit } \\
\hline \multicolumn{5}{|l|}{ Dienstleistungsfreiheit } \\
\hline Kapitalverkehrsfreiheit & $1(\mathrm{DK})$ & & & $2(\mathrm{DK}, \mathrm{GB})$ \\
\hline \multicolumn{5}{|l|}{ Wettbewerb } \\
\hline \multicolumn{5}{|l|}{ Steuerpolitik } \\
\hline \multicolumn{5}{|l|}{ Rechtsangleichung } \\
\hline \multicolumn{5}{|l|}{ Wirtschaftspolitik } \\
\hline Sozialpolitik & $1(\mathrm{~GB})$ & & & \\
\hline \multicolumn{5}{|l|}{ Kohäsion } \\
\hline \multicolumn{5}{|l|}{ Forschung und Technologie } \\
\hline \multicolumn{5}{|l|}{ Umweltpolitik } \\
\hline \multicolumn{5}{|l|}{ Institutionelle Bestimmungen } \\
\hline \multicolumn{5}{|l|}{ Überseeische Territorien } \\
\hline \multicolumn{5}{|l|}{ Finanzielle Bestimmungen } \\
\hline \multicolumn{5}{|c|}{ Allgemeine/Schlussbestimmungen } \\
\hline \multicolumn{5}{|l|}{ Transport } \\
\hline Geldpolitik & $2(\mathrm{DK}, \mathrm{GB})$ & & & $11^{10}$ \\
\hline \multicolumn{5}{|l|}{ Gesundheitspolitik } \\
\hline \multicolumn{5}{|l|}{ Verbraucherschutz } \\
\hline \multicolumn{5}{|l|}{ Transeuropäische Netzwerke } \\
\hline \multicolumn{5}{|l|}{ Industriepolitik } \\
\hline \multicolumn{5}{|l|}{ Entwicklungszusammenarbeit } \\
\hline Außen- und Sicherheitspolitik & $1(\mathrm{DK})$ & & & $1(\mathrm{DK})$ \\
\hline \multicolumn{5}{|l|}{ Justiz und Inneres } \\
\hline Visa, Asyl, Migration & & $3(\mathrm{DK}, \mathrm{EI}, \mathrm{GB})$ & & 3 (DK, EI, GB) \\
\hline Schengen & & $2(\mathrm{EI}, \mathrm{GB})$ & & \\
\hline \multicolumn{5}{|l|}{ Beschäftigungspolitik } \\
\hline Grundrechtecharta & & & & $2(\mathrm{PL}, \mathrm{GB})$ \\
\hline Zivilschutz & & & & \\
\hline Verwaltungszusammenarbeit & & & & \\
\hline Energiepolitik & & & & \\
\hline Tourismus & & & & \\
\hline
\end{tabular}

Anmerkung: Anzahl der neuen Differenzierungen nach Verträgen, Politikfeldern und Ländern. Grau unterlegt sind Politikfelder, die zum jeweiligen Zeitpunkt noch nicht integriert waren

Quelle: Eigene Zusammenstellung.

10 Dänemark (DK), Großbritannien (GB) und Schweden sowie Bulgarien, Estland, Lettland, Litauen, Polen (PL), Rumänien, Tschechische Republik und Ungarn als 2004 beziehungsweise 2007 beigetretene Mitgliedstaaten, die den Euro 2010 noch nicht eingeführt hatten. 
Für jeden dieser Datenpunkte kodieren wir, ob differenzierte Integration vorliegt oder nicht. Dazu aggregieren wir die jährlichen Einträge im EUDIFF1-Datensatz zu ,Differenzierungen'. Eine Differenzierung beginnt in dem Jahr, in dem ein Mitgliedstaat von den Bestimmungen eines Vertragsartikels ausgenommen wird und dauert solange, wie diese Ausnahme ununterbrochen besteht. Gleichzeitige Ausnahmen mehrerer Mitgliedstaaten vom gleichen Vertragsartikel zählen als mehrere Differenzierungen; ebenso die gleichzeitigen Ausnahmen eines Mitgliedstaats von mehreren Vertragsartikeln. Allerdings zählen Differenzierungen, die zum gleichen Politikfeld gehören, den gleichen Mitgliedstaat betreffen und im gleichen Jahr beginnen als eine einzelne Differenzierung. Das hat zwei Gründe. Zum einen resultieren solche Differenzierungen aus einer einzelnen Entscheidung, sind also nicht unabhängig voneinander. Zum anderen würde man ansonsten Entscheidungen, die sich auf zahlreiche Vertragsartikel erstrecken (wie das Schengen-Regime, das bis zu 175 Vertragsartikel betrifft), deutlich höher gewichten als solche, die sich nur auf einen Vertragsartikel beziehen - wie etwa die Einschränkungen der Arbeitnehmerfreizügigkeit, einer zentralen Marktfreiheit, die in nur einem Artikel geregelt ist. ${ }^{11}$

Bei jedem Datenpunkt, der sich durch die Kombination einer Vertragsänderung, eines Politikfelds und eines Mitgliedstaats ergibt, kodieren wir erstens, ob bestehende Differenzierungen vorliegen; zweitens, ob eine neue Differenzierung hinzutritt; und drittens, was der Entstehungszusammenhang dieser Differenzierungen ist (Vertiefung oder Erweiterung). ${ }^{12}$ Neue Differenzierungen sind unser wichtigster Erklärungsgegenstand (siehe Tabelle 1). Informationen über bestehende Differenzierungen benötigen wir zur Prüfung von Hypothese (6) und Informationen über den Entstehungszusammenhang brauchen wir, um eventuelle Störeinflüsse aus den Erweiterungsverträgen auszuschließen. Tabelle 1 zeigt, dass Differenzierung in der Vertragsentwicklung, so wie wir sie hier konzipieren, ein seltenes und auf wenige Politikfelder und Mitgliedsländer konzentriertes Phänomen ist. Bei der Revision der Verträge von Maastricht bis Lissabon wurden nur 29 neue Ausnahmen geschaffen. Sie betreffen nur sieben der 28 Politikfelder und überwiegend drei Mitgliedstaaten: Dänemark, Großbritannien und Irland.

Die anderen Variablen, die wir zur Hypothesenprüfung benötigen, sind die Zugehörigkeit von Politikfeldern zu den staatlichen Kernkompetenzen, die Stärke der nationalen Identität, der Wohlstand der Mitgliedstaaten und die Ratifikation durch Volksabstimmung. Bei den Politikfeldern unterscheiden wir angelehnt an Genschel und Jachtenfuchs Politikfelder, in denen traditionell ein staatliches Monopol bestand, von solchen, in denen das zumindest in liberaldemokratisch-marktwirtschaftlichen Staaten nicht der Fall war. ${ }^{13}$ Solche Monopole erstreckten sich auf die Außen- und Verteidigungspolitik, die Innenpolitik, die Justiz- und Rechtspolitik, die Geldpolitik, die Fiskalpolitik und die öffentliche Verwaltung. In Tabelle 1 fallen darunter: Geldpolitik, Steuerpolitik, Außen- und Sicherheitspolitik, Justiz und Inneres, Visa-, Asyl- und Migrationspolitik, Schengen, Grundrechtecharta und Verwaltungspolitik.

11 Schimmelfennig/Winzen: Instrumental and Constitutional Differentiation, 2014.

12 Durch diese Messung erfassen wir keine Differenzierungen, die nicht mit einem der genannten Verträge beginnen und bereits vor dem Inkrafttreten der folgenden Vertragsänderungen enden. Zu beachten ist auch, dass wir das Schengener Übereinkommen erst mit dem Vertrag von Amsterdam in den Datensatz aufnehmen (es war bereits zuvor in Kraft, wurde aber erst mit diesem Vertrag in das Gemeinschaftsrecht übernommen).

13 Philipp Genschel/Markus Jachtenfuchs (Hrsg.): Regulatory or Federal? The European Integration of Core State Powers, Oxford 2013; vgl. auch Frank Schimmelfennig/Dirk Leuffen/Berthold Rittberger: The European Union as a System of Differentiated Integration: Interdependence, Politicization and Differentiation, unveröffentlichtes Manuskript, 2014. 
Hooghe und Marks zeigen, dass Europaskepsis mit exklusiven nationalen Identitäten zusammenhängt, während sich multiple Identitäten, die sowohl eine nationale als auch eine europäische Identität einschließen, nicht systematisch negativ auf die Zustimmung zur europäischen Integration auswirken. ${ }^{14}$ Wir operationalisieren die Identitätsvariable deshalb als den Anteil von Befragten mit einer ausschließlich nationalen Identität pro Mitgliedstaat. Zur Messung ziehen wir in Übereinstimmung mit Hooghe und Marks die Eurobarometer-Umfragen heran, in denen gefragt wird, ob eine Person sich in naher Zukunft ausschließlich als Angehörige(-r) der eigenen Nation sieht oder als Europäer(-in) oder beides. Wir verwenden jeweils die Umfragen im Vorfeld einer Vertragsrevision: die Jahre 1992 und 1993 für den Vertrag von Maastricht, die Zeit von Dezember 1995 bis Mai 1998 für den Vertrag von Amsterdam, die Umfragen aus den Jahren 2000 und 2001 für den Vertrag von Nizza und die Umfragen der Jahre 2004, 2005 und 2010 für den Vertrag von Lissabon. ${ }^{15}$

Wohlstand messen wir als Bruttosozialprodukt pro Kopf in US-Dollar (kaufkraftbereinigt) nach Angaben der Weltbank (World Development Indicators) jeweils für das Jahr des Inkrafttretens der Verträge. Für die Ratifikation kodieren wir für jedes Land und jeden Vertrag, ob eine Volksabstimmung stattgefunden hat.

\section{Analyse}

Wir analysieren die Zusammenhänge zwischen differenzierter Integration, exklusiver Identität, staatlichen Kernkompetenzen und Wohlstand zunächst deskriptiv. Schaubild 2 legt nahe, dass in den Vertragsreformen seit Maastricht vor allem solche Länder neue Differenzierungen erzielt haben, die eine stark ausgeprägte nationale Identität aufweisen. Dänemark und Großbritannien, die von zahlreichen vertragsrechtlich geregelten Politikbereichen ausgenommen sind, sind hier die eindrücklichsten Beispiele. Ein vergleichbares Schaubild lässt allerdings keinen nennenswerten Zusammenhang zwischen Wohlstand und differenzierter Integration erkennen (hier nicht gezeigt). Ein erster Blick auf unsere Daten zeigt des Weiteren, dass der Großteil neuer Differenzierungen Politikbereiche betrifft, die wir als staatliche Kernkompetenzen betrachten. So finden wir insgesamt 25 Differenzierungen in diesen und nur vier in anderen Politikbereichen. Diese vier Fälle beinhalten die (temporäre) britische Ablehnung der sozialpolitischen Integration während der 1990er Jahre sowie drei Ausnahmen im Bereich Kapitalverkehrsfreiheit.

In einem zweiten Schritt testen wir mittels einer logistischen Regressionsanalyse, inwiefern unsere erklärenden Variablen die Wahrscheinlichkeit beeinflussen, dass ein Mitgliedstaat in einer Vertragsreform und einem Politikbereich eine Ausnahme erzielt. Man muss in dieser Analyse beachten, dass wir bei 1.893 Kombinationen aus Vertragsreformen, Politikbereichen und Ländern nur 29 Fälle neuer Differenzierung haben (1,5 Prozent). Vertragsrechtliche Ausnahmen sind also in jedem Fall ein seltenes Ereignis. Ziel unserer Untersu-

14 Liesbet Hooghe/Gary Marks: Calculation, Community, and Cues. Public Opinion on European Integration, in: European Union Politics 4/2005, S. 419-443.

15 Im Vorfeld des Vertrages von Lissabon wurde die Frage nach der Identität nicht gestellt. 2004 und 2005 erscheinen plausibel, weil der Inhalt des Vertrags im Wesentlichen mit dem gescheiterten Verfassungsvertrag übereinstimmt. Die Daten wurden der interaktiven Eurobarometer-Webseite entnommen. Vgl. Europäische Kommission: Eurobarometer surveys. In the near future do you see yourself as...?, abrufbar unter: http://ec.eu ropa.eu/public_opinion/cf/showchart_column.cfm?keyID=266\&nationID $=11,1,27,28,17,2,16,18,13,6,3,4,22$, $7,8,20,21,9,23,24,12,19,29,26,25,5,14,10,30,15$,\&startdate $=1992.04 \&$ enddate $=2010.06 \&$ lang $=$ de (letzter Zugriff: 13.3 .2014$)$. 
chung ist festzustellen, ob die in den Hypothesen benannten Länder- und Politikfeldmerkmale die geringe Grundwahrscheinlichkeit einer Differenzierung erhöhen.

\section{Schaubild 2: Exklusive Identität und neue Differenzierungen}

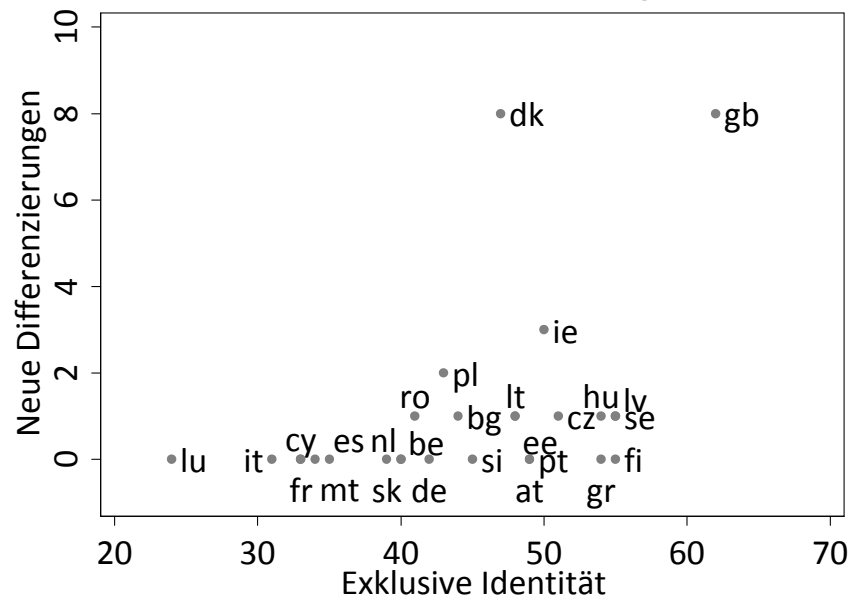

Anmerkung: Belgien (be), Bulgarien (bg), Dänemark (dk), Deutschland (de), Estland (ee), Finnland (fi), Frankreich (fr), Griechenland (gr), Irland (ie), Italien (it), Lettland (lv), Litauen (lt), Luxemburg (lu), Malta (mt), Niederlande (nl), Österreich (at), Polen (pl), Portugal (pt), Rumänien (ro), Schweden (se), Slowakei (sk), Slowenien (si), Spanien (es), Tschechische Republik (cz), Ungarn (hu), Vereinigtes Königreich (gb), Zypern (cy).

Quelle: Eigene Darstellung.

Tabelle 2 zeigt die Ergebnisse von fünf logistischen Regressionen. ${ }^{16}$ In Modell 1 konzentrieren wir uns auf die Stärke der nationalen Identität und staatliche Kernkompetenzen. In den folgenden Modellen fügen wir einen Interaktionseffekt, unseren Wohlstandsindikator, eine Variable für bestehende Differenzierungen und eine für Volksabstimmungen hinzu. Wichtig ist, dass die logistische Regression auf anderen Annahmen und einer anderen Schätzmethode als die klassische lineare Regression basiert. Dieser Ansatz erlaubt es uns, die Wahrscheinlichkeit eines ,positiven` Ergebnisses zu schätzen, erschwert jedoch die Interpretation der Resultate. So können wir aus Tabelle 2 zwar entnehmen, welche Variablen die Wahrscheinlichkeit, dass eine neue Differenzierung zustande kommt, signifikant positiv oder negativ beeinflussen. Die Regressionskoeffizienten selbst, die in einer linearen Regression das substanzielle Ausmaß der statistisch signifikanten Effekte unmittelbar anzeigen, lassen sich in der logistischen Regression aber erst nach weiteren Transformationen inter-

16 Um die Erklärungskraft der Modelle zu bewerten, überprüfen wir, wie viele unserer Fälle die Modelle richtig klassifizieren (als ,cut-off' Punkt wählen wir den Anteil tatsächlich beobachteter Differenzierungen in unserer Gesamtbevölkerung). Modell 1 klassifiziert 80,7 Prozent der Fälle korrekt, Modell 2 78,5 Prozent, Modell 381 Prozent, Modell 4 81,2 Prozent und Modell 5 81,6 Prozent. Ebenfalls haben wir überprüft, ob die Signifikanz der Ergebnisse davon abhängt, dass wir auch erweiterungsbasierte Differenzierungen als neue Differenzierungen betrachten. Das ist nicht der Fall, mit Ausnahme von Modell 3 (siehe Text). Die Ergebnisse bleiben stabil, wenn wir die jeweiligen Vertragszeitpunkte mit Dummy-Variablen identifizieren. Wir haben auch drei ,volle 'Modelle berechnet: eines mit allen Variablen außer Wohlstand, eines mit allen Variablen und eines mit allen Variablen und den Restriktionen von Modell 3 (siehe Text). Diese Modelle erhöhen unsere Erklärungskraft nur geringfügig. Sie bestätigen die Ergebnisse mit der Anmerkung, dass der Identitätseffekt das .1 Signifikanzniveau einmal knapp erreicht und zwei Mal knapp verfehlt. 
pretieren. Entsprechend der Empfehlungen in der Literatur, konzentrieren wir uns in der folgenden Diskussion darauf, was die Regressionskoeffizienten für die Wahrscheinlichkeit, dass eine neue Differenzierung zustande kommt, bedeuten. ${ }^{17}$ Wir verzichten auf eine technische Erläuterung der Herleitung der Wahrscheinlichkeiten, die wir in der Folge besprechen, betonen jedoch, dass diese sich aus den Regressionskoeffizienten in Tabelle 2 ergeben.

Tabelle 2: Logistische Regressionsmodelle

\begin{tabular}{lccccc}
\hline & Modell 1 & Modell 2 & Modell 3 & Modell 4 & Modell 5 \\
\hline Kernkompetenz & $3.1(.39)^{*}$ & $4.44(2.56)^{\circ}$ & $2.7(.27)^{*}$ & $2.77(.52)^{*}$ & $3.13(.39)^{*}$ \\
Identität & $.09(.03)^{*}$ & $.11^{\circ}(.06)$ & $.09(.04)^{*}$ & $.06(.03)^{*}$ & $.09(.03)^{*}$ \\
IdentitätKernkompetenz & & $-.03(.04)$ & & & \\
Bruttosozialprodukt/Kopf & & & $-.00(.00)^{*}$ & & \\
Bestehende Diff. & & & $3.01(.07)^{*}$ & \\
Referendum & & & & $1.09(.41)^{*}$ \\
Konstante & -9.94 & -11.09 & -12.94 & -9.03 & $-10.13(.41)^{*}$ \\
& $(1.61)^{*}$ & $(3.43)^{*}$ & $(2.28)^{*}$ & $(1.29)^{*}$ & 1893 \\
$\mathrm{~N}$ & 1893 & 1893 & 1787 & 1893 & 189 \\
Log-pseudolikelihood & -115.57 & -115.49 & -88.54 & -94.08 & -113.24 \\
\hline
\end{tabular}

Anmerkung: $* p<.05,{ }^{\circ} p<.1$. Standardfehler (,country-clustered ') in Klammern. Modell 3 hat eine geringere Fallzahl, weil Luxemburg als extremer , Outlier 'bei der Variable BSP von der Analyse ausgenommen ist.

Quelle: Eigene Darstellung.

\section{Schaubild 3: Exklusive Identität und die Wahrscheinlichkeit neuer Differenzierungen}

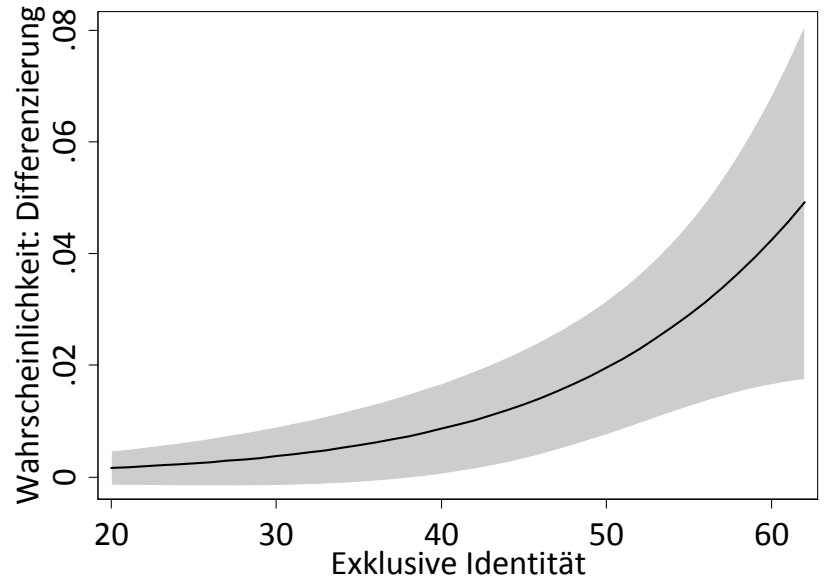

Anmerkung: Der grau unterlegte Bereich zeigt die Konfidenzintervalle.

Quelle: Eigene Darstellung.

17 Wir berechnen Wahrscheinlichkeiten und Konfidenzintervalle mit STATAs „,margins“ Befehl. Falls nicht anders beschrieben, berechnen wir die Wahrscheinlichkeit für eine gegebene Variable als Durchschnitt über alle Werte der anderen Variablen. Die „Margins“-Methode Konfidenzintervalle zu berechnen, kann zu Wahrscheinlichkeiten unter null führen. In solchen Fällen haben wir die Konfidenzintervalle mit Simulationen unter Zuhilfenahme der „Clarify“ Software überprüft. Vgl. Michael Tomz/Jason Wittenberg/Gary King: CLARIFY: Software for Interpreting and Presenting Statistical Results. Version 2.1, Stanford University/University of Wisconsin/ Harvard University 2003. 
Die erste Hypothese geht davon aus, dass die Integration staatlicher Kernkompetenzen mit höherer Wahrscheinlichkeit von Differenzierungen betroffen ist als andere Politikfelder. Dieser Effekt bestätigt sich in Modell 1. Die Wahrscheinlichkeit, dass eine neue Differenzierung in einem kernstaatlichen Bereich auftritt liegt bei 5,6 Prozent und somit deutlich über der Häufigkeit von Differenzierungen in der Grundgesamtheit. In anderen Politikbereichen liegt die Wahrscheinlichkeit einer Differenzierung bei verschwindend geringen 0,3 Prozent. Dieser Effekt bestätigt sich statistisch wie substanziell in den weiteren Modellen, mit der Ausnahme von Modell 2, das wir in Kürze besprechen. Die zweite Hypothese bringt Differenzierungen mit einer ausgeprägten nationalen Identität in Verbindung. Auch dieser Effekt findet sich in Modell 1. Wie Schaubild 3 zeigt, liegt die Differenzierungswahrscheinlichkeit in Ländern mit einer inklusiven Identität nahe null. Sie steigt auf über vier Prozent in Mitgliedstaaten mit stark ausgeprägten nationalen Identitäten.

Wir vermuten ebenfalls einen Zusammenhang zwischen den ersten beiden Hypothesen: Eine stark ausgeprägte Identität wirkt sich besonders bei der Integration staatlicher Kernkompetenzen aus. Diese Vermutung bestätigt sich zunächst nicht. Der Interaktionseffekt in Modell 2 ist nicht signifikant. Zudem zeigen Diagnosetests, dass Modell 2 keine verbesserte Erklärungskraft gegenüber Modell 1 bringt. Wir können daher sagen, dass sich nationale Identität grundsätzlich in staatlichen Kernbereichen nicht anders auswirkt als in anderen Politikfeldern.

\section{Schaubild 4: Exklusive Identität und staatliche Kernkompetenzen}

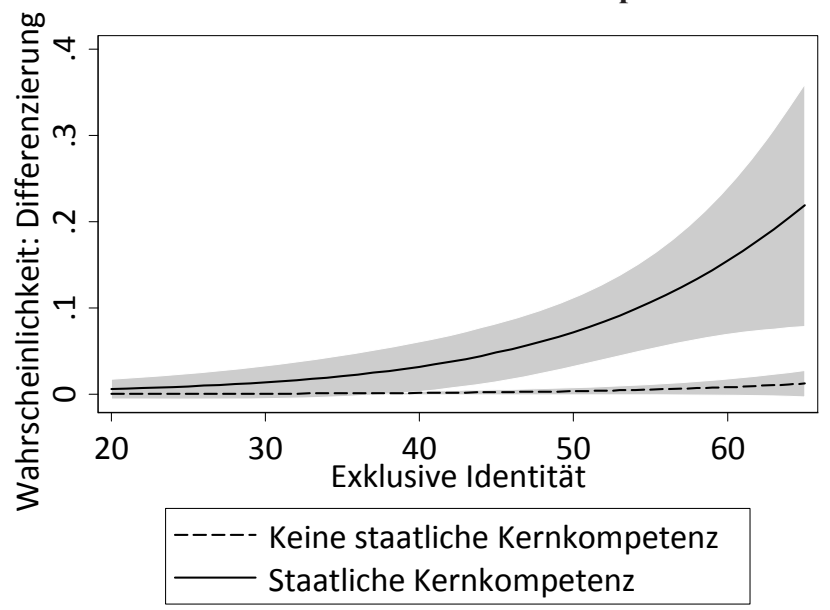

Anmerkung: Der grau unterlegte Bereich zeigt die Konfidenzintervalle.

Quelle: Eigene Darstellung.

In logistischen Regressionsmodellen können sich jedoch trotz eines statistisch insignifikanten Interaktionseffektes Interaktionen zwischen Variablen ergeben. ${ }^{18}$ In der Tat sehen wir in Schaubild 4, welches auf den Ergebnissen aus Modell 1 beruht, dass eine ausgeprägte nationale Identität die Wahrscheinlichkeit einer neuen Differenzierung nur in staatlichen Kernbereichen stark erhöht. Während die Chance, dass ein Land mit einer stark exklusiven

18 William D. Berry/Jacqueline H. R. DeMeritt/Justin Esarey: Testing for Interaction in Binary Logit and Probit Models: Is a Product Term Essential?, in: American Journal of Political Science 1/2010, S. 248-266. 
Identität eine neue Ausnahme in einem solchen Bereich erzielt bei bis zu circa 20 Prozent liegt, ist sie in anderen Bereichen nicht von null zu unterscheiden. Ebenfalls können wir im Umkehrschluss sagen, dass die Unterscheidung zwischen staatlichen Kern- und anderen Bereichen für Länder mit einer sehr inklusiven Identität keine praktische Relevanz hat.

Es ist wichtig zu betonen, dass diese Erkenntnisse nicht bedeuten, dass Identität sich in bestimmten Politikbereichen grundsätzlich anders auswirkt als in anderen; oder dass die Unterscheidung zwischen Politikbereichen für Länder mit inklusiven Identitäten grundsätzlich anders wirkt als für andere. Vielmehr sehen wir in Schaubild 4 die Folgen der sehr geringen Grundwahrscheinlichkeit, dass überhaupt eine Differenzierung zustande kommt. Wenn wir es mit einem wenig zentralen Politikbereich zu tun haben, ist diese Wahrscheinlichkeit bereits so gering, dass selbst starke Effekte unserer Identitätsvariable in absoluten Zahlen unbedeutend werden. Beispielsweise sehen wir der gestrichelten Linie in Schaubild 4 nicht an, dass die Differenzierungswahrscheinlichkeit von unter 0,03 Prozent um das 44fache auf 1,2 Prozent steigt. Statistiker sprechen hier von einem „Kompressionseffekt" ${ }^{19}$ Wir schließen daraus, dass es einer Kombination mehrerer Faktoren bedarf, damit sich die Wahrscheinlichkeit einer neuen vertragsrechtlichen Ausnahmeregel substanziell erhöht.

Hypothese 3 geht davon aus, dass wohlhabende Mitgliedstaaten eher an der differenzierten Integration beteiligt sind als weniger wohlhabende. Wie die deskriptive Betrachtung unserer Daten bereits nahelegte, zeigt sich dieser Effekt in der logistischen Regressionsanalyse nicht, wenn wir mit derselben Grundgesamtheit arbeiten, die auch den anderen Modellen zugrunde liegt. Diese Grundgesamtheit beinhaltet jedoch acht neue Differenzierungen, die die relativ armen Staaten der mittel- und osteuropäischen Erweiterungsrunde im Lissaboner Vertrag erhielten und die unmittelbar aus der zum Beitritt vereinbarten verzögerten Teilnahme an der Währungsunion folgten. Wenn wir diese Differenzierungen von der Analyse ausnehmen, ergibt sich ein statistisch signifikanter Wohlstandseffekt (siehe Modell 3 in Tabelle 2). Schaubild 5 zeigt, dass die Differenzierungswahrscheinlichkeit in Folge wachsenden Wohlstands von etwa null auf deutlich über 2 Prozent ansteigt.

Die Hypothesen 4 und 5 beschreiben die Folgen von Volksabstimmungen und bestehender Differenzierung. In der Tat finden wir in Modell 4, dass eine bestehende Differenzierung die Wahrscheinlichkeit einer neuen Differenzierung von null auf zehn Prozent erhöht. Weitere Analysen von Modell 4 (hier nicht gezeigt) legen nahe, dass zwischen bestehenden Differenzierungen und einer exklusiven nationalen Identität eine vergleichbare Beziehung wie zwischen staatlichen Kernbereichen und Identität besteht (siehe Schaubild 3). Diese Beziehung ist jedoch aufgrund des seltenen Auftretens bestehender Differenzierungen mit größerer Unsicherheit behaftet und nur teilweise statistisch signifikant. Sie untermauert dennoch die Erkenntnis, dass Differenzierungen besonders durch das Auftreten mehrerer Risikofaktoren wahrscheinlich werden. Modell 5 zeigt, dass Differenzierungen eher auftreten, wenn Länder Verträge per Volksabstimmung ratifizieren. In diesem Fall erhöht sich die Wahrscheinlichkeit einer Ausnahmeregelung von 1 auf 3,4 Prozent.

19 Ebenda, S. 251-252. 


\section{Schaubild 5: Wohlstand und differenzierte Integration}

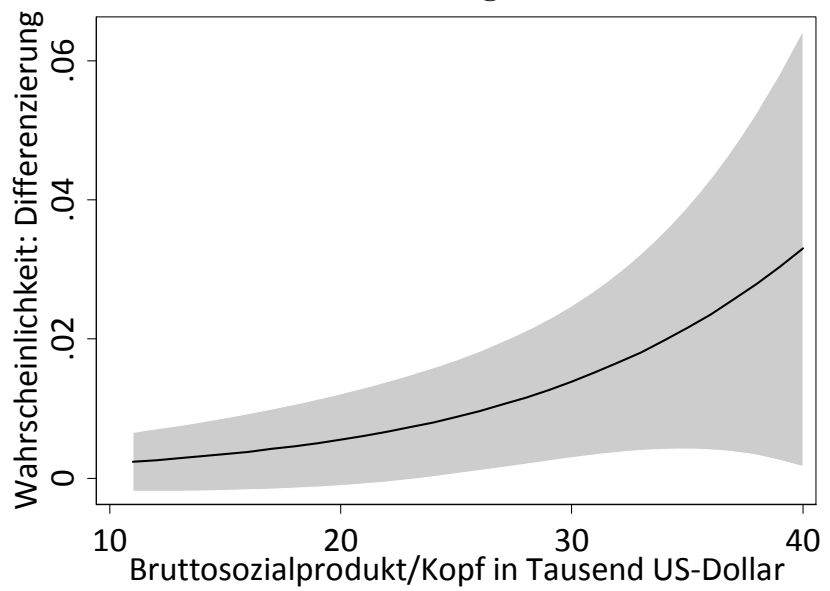

Anmerkung: Der grau unterlegte Bereich zeigt die Konfidenzintervalle.

Quelle: Eigene Darstellung.

Zusammenfassend stellen wir fest, dass differenzierte Integration in staatlichen Kernkompetenzen (Hypothese 1), für Länder mit exklusiver nationaler Identität (Hypothese 2) und großem Wohlstand (Hypothese 3), bei Ratifizierung per Volksabstimmung (Hypothese 5) und im Falle bestehender Differenzierungen (Hypothese 6) überdurchschnittlich wahrscheinlich ist. Wir finden keine Interaktion zwischen Identität und staatlichen Kernkompetenzen (Hypothese 4). Allerdings zeigt die Analyse, dass insbesondere Kombinationen mehrerer Faktoren die Differenzierungswahrscheinlichkeit stark erhöhen. Umgekehrt kann bereits die Abwesenheit eines Faktors diese Wahrscheinlichkeit so stark senken, dass Varianz in anderen Faktoren keine praktische Auswirkung mehr hat.

\section{Schluss: Eine dauerhafte Kern-Peripherie-Struktur in der europäischen Integration}

Seit den 1990er Jahren wurde die Vertiefung der europäischen Integration von ihrer Differenzierung begleitet. Unsere Analyse zeigt, dass dieser Prozess durch das Ausgreifen der europäischen Integration auf staatliche Kernkompetenzen und die Identitätsunterschiede der Mitgliedstaaten erklärt werden kann. Wir interpretieren die Ergebnisse so, dass Mitgliedsgesellschaften mit vergleichsweise ausgeprägter nationaler Identität befürchten, dass die Vertiefung der europäischen Integration die Autonomie des von ihnen geschätzten Nationalstaats untergräbt. Sie streben daher Ausnahmen von der Vertiefung an, vor allem, wenn sie darauf abzielt, Kernkompetenzen des Nationalstaats europäisch zu integrieren. Die Ergebnisse sind außerdem mit der Annahme vereinbar, dass wohlhabendere Staaten sich eine solche autonomieschonende Differenzierungspolitik am ehesten leisten können und dass eine Ratifikation durch Volksabstimmung die Identitätspolitik stärkt. Damit decken sich die Resultate mit Grundannahmen der konstruktivistischen oder postfunktionalistischen Integrationstheorie über die ideellen Voraussetzungen und Hemmnisse europäischer Integration. ${ }^{20}$

20 Liesbet Hooghe/Gary Marks: A Postfunctionalist Theory of European Integration: From Permissive Consensus to Constraining Dissensus, in: British Journal of Political Science 1/2009, S. 1-23. 
Ökonomische Präferenzen und Kapazitäten scheinen für die differenzierte Integration in der Vertragsentwicklung keine wesentliche Rolle zu spielen.

Aus diesem Befund ergeben sich zwei weitere Schlussfolgerungen. Zum einen erscheint differenzierte Integration als probates Instrument, um auf unterschiedlich gelagerte nationale Befindlichkeiten Rücksicht zu nehmen. Sie erlaubt es, auf Mitgliedsgesellschaften mit ausgeprägter nationaler Identität und nationalem Autonomiebedürfnis Rücksicht zu nehmen, ohne die anderen Mitglieder daran zu hindern, mit der Vertiefung der Integration voranzuschreiten. Zum anderen lässt die Verwurzelung der differenzierten Integration in der nationalen Identität erwarten, dass differenzierte Integration von hoher Stabilität geprägt ist. Nationale Identitäten haben sich als hochgradig resistent gegenüber dem Fortschritt der institutionellen Integration erwiesen. Gleiches gilt für die Identitätsunterschiede zwischen den Mitgliedstaaten. Sie lassen weiterhin den Schluss zu, dass es sich bei der differenzierten Integration in der Vertragsentwicklung nicht einfach um ein ,Europa der zwei Geschwindigkeiten' handelt, in dem die Nachzügler nach einiger Zeit zu den Vorreitern aufschließen werden, sondern um eine dauerhafte Unterteilung in ein ,Kerneuropa' aus integrationsfreundlicheren und eine ,Peripherie` aus stärker autonomie- und identitätsbewussten Gesellschaften.

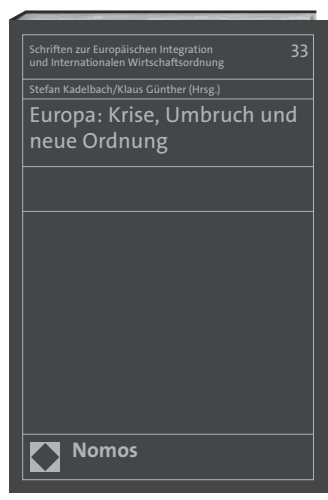

\section{Europa: Krise, Umbruch und neue Ordnung}

Herausgegeben von Stefan Kadelbach und Klaus Günther

2014, 199 S., brosch., 52,-€

ISBN 978-3-8487-1385-1

(Schriften zur Europäischen Integration und Internationalen Wirtschaftsordnung, $B d$. 33)

www.nomos-shop.de/22655

Die Staatsschuldenkrise in der EU hat nicht nur eine stark ausgeprägte wirtschaftsrechtliche Dimension, sondern sie zeigt auch strukturelle Probleme der Union auf. Die Staatsschuldenkrise wird in diesem Band mit Beiträgen aus Perspektiven der Geschichts-, Wirtschafts-, Rechts- und Politikwissenschaften sowie der Sozialphilosophie in Hinblick auf die politische Kultur der Union dargestellt.

Portofreie Buch-Bestellungen unter www.nomos-shop.de

Alle Preise inkl. Mehrwertsteuer

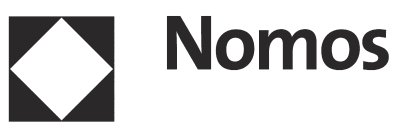

